\title{
The performance of a magnetorheological fluid in squeeze mode
}

\begin{abstract}
In a magnetorheological (MR) fluid, the rheological properties can be changed in a controlled way, the changes being reversible and dependent on the strength of a magnetic field. The fluids have potentially beneficial applications when placed in various geometrical arrangements. The squeeze mode is a geometric arrangement where two flat parallel solid surfaces, facing each other, are pushed towards each other by an external force, operating at right angles to the surfaces. The liquid initially in the gap between them is free to move away from this increasingly small gap, and it does so by flowing parallel to the surfaces, and collecting in a region where it is no longer in the gap between them. The performance of an MR fluid in compression (squeeze) mode has been studied with the magnetic field being generated by a coil carrying different magnitudes of DC electrical current. A test rig was designed to perform this operation with the flat surfaces being horizontal and being pushed together in a vertical direction and the liquid being forced to move in all directions in a horizontal plane. The rig operated by decreasing the size of the gap at a constant rate. For each trial the current in the coil was kept constant and the instantaneous compressive force was recorded. When plotting compressive stress against compressive strain for each trial, the slope of the curve was found to be larger in general when the current was larger. This was an expected result; however the behaviour is more complicated than this. For a significant range of values of the compressive strain, the slope falls to zero, so that the compressive stress shows no increase during this period, while the compressive strain continues
\end{abstract}


The performance of a MR fluid in squeeze mode

to increase. The details of this behaviour are strongly dependent on the initial size of the gap.

\section{Introduction}

A magnetorheological (MR) fluid is a suspension of micron-sized magnetically soft particles in a carrier liquid, which exhibits dramatic changes in rheological properties. The change from a solid-like state to a free-flowing liquid state is reversible and depends on the presence of a magnetic field. Under the influence of a magnetic field these particles arrange themselves to form very strong chains of "fluxes" (Hagenbuchle and Liu 1997), with a pole of one particle being attracted to the opposite pole of another particle. Once aligned in this manner, the particles are restrained from moving away from their respective flux lines and act as a barrier preventing the flow of the carrier fluid.

Potential applications of MR fluids have been suggested especially applications in automotive industry where benefits could be achieved in parts such as clutches, brakes, dampers and actuators (Jolly et al 1999, Huang et al 2002, Yoo and Wereley 2002). Most of the researchers assume a geometrical arrangement referred to as shear mode in their design but they found that the magnitudes of the stress in systems with shear mode geometry are too low to be of value in the potential applications listed above. However, the geometrical arrangement designated squeeze mode can produce compression and tensile stresses which are much higher and this has generated new interest in this approach. The values of compressive stress are similar to those reported in experimental studies using electrorheological (ER) fluids (Lukkarinen and Kaski 1998, Monkman 1995, Tian et al 2002a). Tian et al (2002b) have investigated the stepwise compression of fluids containing zeolites and silicones in squeeze mode. They also studied the compression starting with different initial gap sizes and under different applied electrical potentials (Tian et al 2003). 
The performance of a MR fluid in squeeze mode

Despite the fact that MR fluids have been investigated repeatably with the squeeze mode geometry, many of the studies dealt with controlling vibration for rotor systems. Forte et al (2004), Ahn et al (2004) and Carmignani et al (2006) developed an MR squeeze film damper system for rotor applications. Wang et al (2005) investigated the dynamic performance of this system and subsequently (Wang et al 2006) analyzed the mechanical properties of the film and the unbalanced response characteristics of the MR squeeze film in the damper-rigid rotor system. However, a thorough study of the stress-strain characteristics in compression of MR fluids in squeeze mode is still not complete. This paper presents an experimental investigation of the performance of an MR fluid in squeeze mode under different applied currents and starting with different gap sizes.

\section{Design of magnetic circuit}

The main objective of the magnetic circuit design is to produce the correct magnetic flux density across the MR fluid. A length of cooper wire with a resistance of 29 ohms was wound around a cylinder, forming 2750 turns, in order to generate a magnetic field. The magnetic behaviour of the equipment was analysed using a Finite Element Method Magnetics (FEMM) software package (Meeker 2006). The magnetic properties of the nonmagnetic materials were assumed to be linear whereas the properties of magnetic materials such as low carbon steel were assumed to follow the $B-H$ curves given in the software package. An MR fluid of type MRF-241ES (water-based) produced by the Lord Corporation was tested in these experiments. The typical magnetic properties for this material are shown in figure 1.

An axial symmetric model was selected in the FEMM software package. The magnetic flux density distribution within the fluid is shown in figure 2 . The following parameters were used to specify the geometry of the coil; inner diameter $=60 \mathrm{~mm}$, outer diameter $=92 \mathrm{~mm}$, and width $=100 \mathrm{~mm}$. These parameters limit the area in which the copper wire can be 
The performance of a MR fluid in squeeze mode

wound. The current density was limited to $4 \mathrm{Amm}^{-2}$ in connection with the appropriate wire diameter.

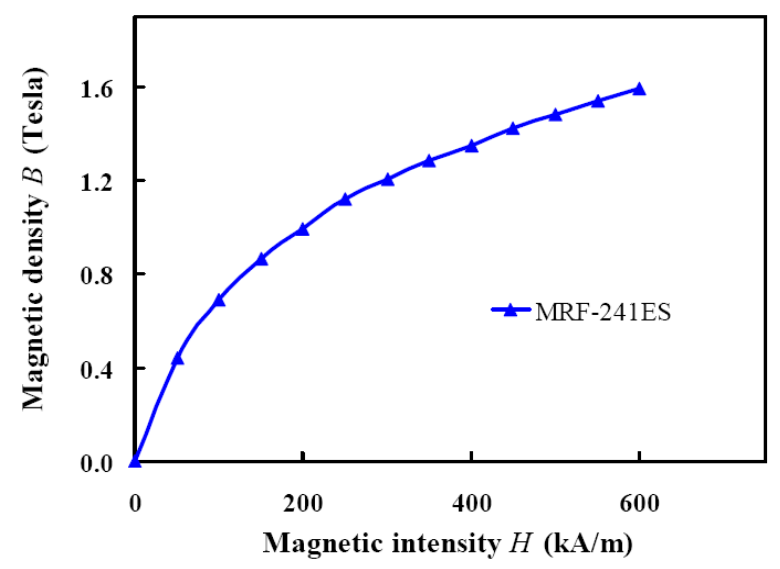

Figure 1. Magnetic induction curve for MRF-241ES (Lord 2007).

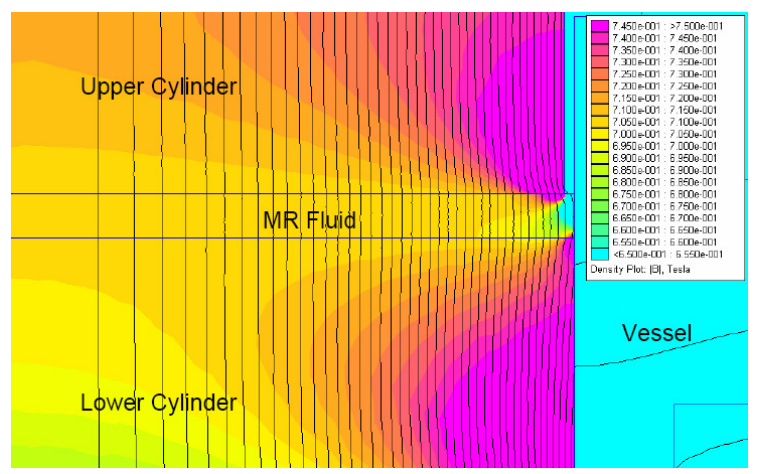

Figure 2. Flux density B, which is about 0.7 Tesla is distributed evenly inside the MR fluid when a of 1.6 Amps current flow through the coil, and the gap size is set to $2 \mathrm{~mm}$. Two-dimensional flux lines seemed to penetrate the MR fluid and aligned to the compression direction.

\section{Experiment}

A schematic diagram of the experimental set-up, similar to that used for studying ER fluid by Tian et al (2003) is as shown in figure 3. The diameter of the vessel used to contain the MR fluid is a little larger than the diameter of the upper cylinder, so that the gap region 
The performance of a MR fluid in squeeze mode

remains flooded by the MR fluid throughout the compression. The compression of the MR fluid was carried out by lowering the upper cylinder towards the bottom of the vessel containing the fluid using a computer-controlled movement. Two sets of experimental trials have been carried out. In the first set, the gap size was set initially to $2 \mathrm{~mm}$ and different current values $(0.4,0.8,1.2$ and 1.6 Amps) were maintained throughout the compression trial. Then, the upper cylinder was lowered at a speed of $0.5 \mathrm{~mm} / \mathrm{min}$, which can be considered as quasi-static loading and the load was recorded continuously. The same procedure was carried out in the set of experimental trials, except that the initial gap size between the upper cylinder and the bottom of the vessel was set to $1 \mathrm{~mm}$.

A DC Magnetometer (gauss-meter) supplied by AlphaLab Inc. was used to validate the results of the FEMM software package for the magnetic field strength generated by the coil. Figure 4 shows the magnitude of the magnetic field strength (Oersted) for both the measured and the simulated results at an initial gap size of $2 \mathrm{~mm}$.

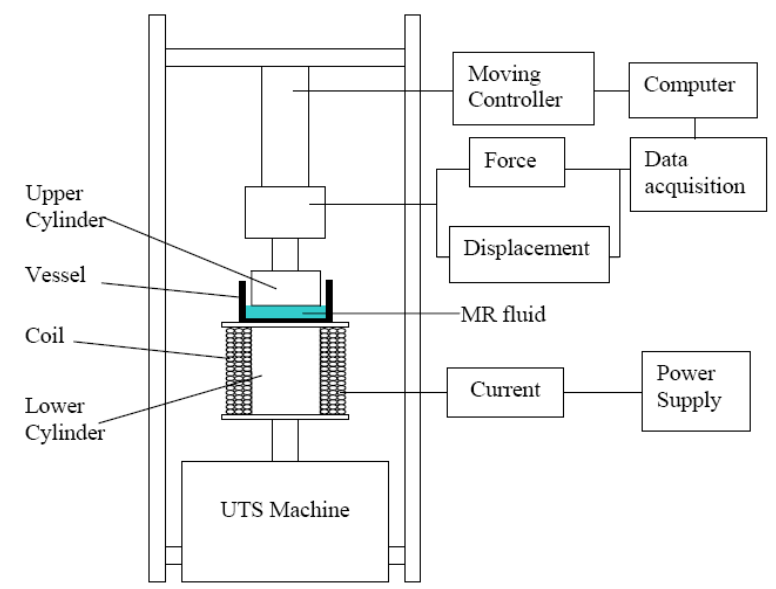

Figure 3. The bottom of the upper cylinder and the bottom of the vessel containing the MR fluid are two parallel surfaces. Measured amount of fluid was sandwiched between these two parallel surfaces, so that the fluid was compressed in a direction normal to these surfaces when the upper cylinder moved towards the bottom of the vessel containing the fluid. All experiments were performed in a displacement control mode. 
The performance of a MR fluid in squeeze mode

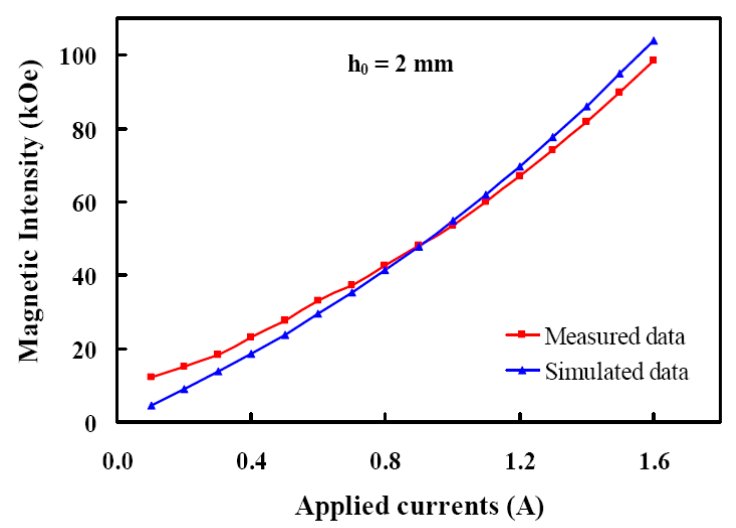

Figure 4. Values of the magnetic field strength obtained by direct measurement and simulation are in good agreement. The magnetic field strength increased when the applied current increased.

\section{Results and discussion}

The relationship between the compressive stress and the compressive strain under different applied currents is depicted in figures 5(a) and 5(b). The compressive stress increases with increasing compressive strain.

Applying Kirchhoff's law for magnetic circuits, the number of turns $N$ (in a rail) and applied current $I$ determine the magnitude of the magnetic field strength $H$.

$$
N I=\oint H . d l
$$

In this equation, length $l$ refers to the total length of the whole magnetic circuit and must include the length of the lower cylinder around which the coil is wound. Therefore, for the same number of turns, a higher electrical current or a small gap size will result in a great magnetic field strength.

In view of this one might have expected that the compressive stress can be influenced by the applied magnetic field strength but in fact higher values of compressive stresses are required when the initial gap size is set to $2 \mathrm{~mm}$ in comparison to the situation when the initial gap size of $1 \mathrm{~mm}$ has been set as shown in figure 6 . This result emphasizes that the 
The performance of a MR fluid in squeeze mode

compressive stress of MR fluid is strongly affected by the initial gap size which is similar to that reported by Tian et al (2003).

In another observation, for a constant compressive speed at $0.5 \mathrm{~mm} / \mathrm{min}$, the value of the compressive stress increases as the gap closes (figure 7). The curves show greater values of compressive stress as at the gap between the two cylinders become smaller. However, all the curves show the same values of compressive stress at the beginning of compression until the instantaneous gap size has been reached nearly $1.8 \mathrm{~mm}$. Then, the curves show a variation according to the current.

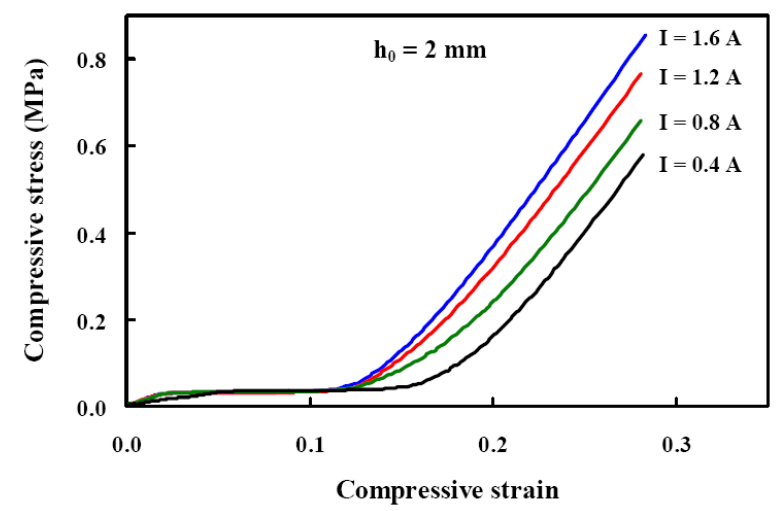

(a)

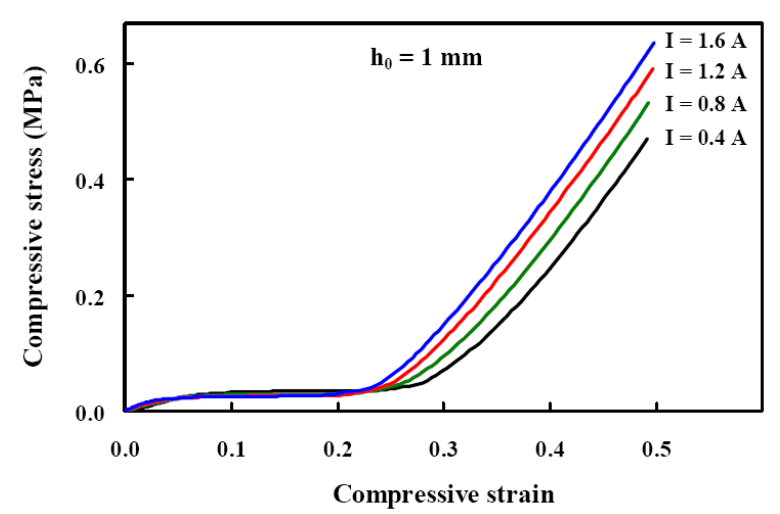

(b)

Figure 5. Compressive stress versus compressive strain under different applied currents for $(a) 1$ $\mathrm{mm}$ of the initial gap size and $(b) 2 \mathrm{~mm}$ of the initial gap size. 
The performance of a MR fluid in squeeze mode

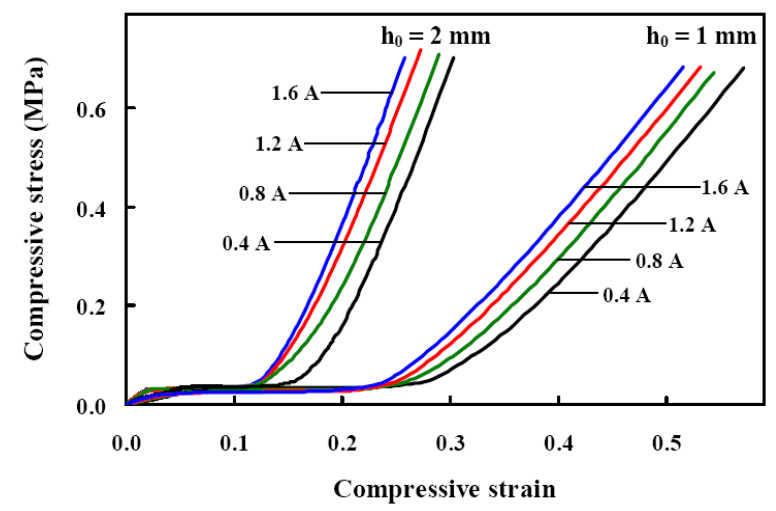

Figure 6. Comparison between two different initial gap sizes (1 and $2 \mathrm{~mm}$ ) under various applied currents during the process of compression.

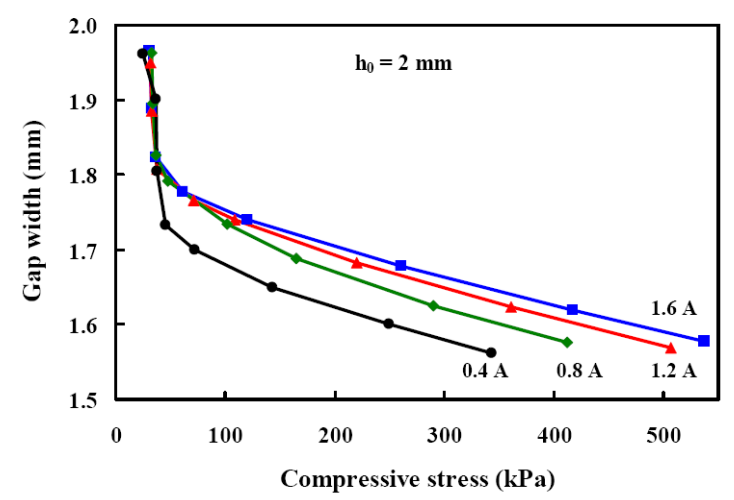

Figure 7. Gap width distances as a function of compressive stress at different applied currents ranging from 0.4 to 1.6 Amps.

The process of compression of the MR fluid always shows the same three regions. All the curves of compressive stress versus compressive strain show similar characteristics despite the fact that the applied current ranged between 0.4 to 1.6 Amps and, the initial gap size varied between 1 and $2 \mathrm{~mm}$. Figure 8 shows the results for the process of compression at a larger scale. In the first region, the compressive stress increased gradually with the increase of the compressive strain until it reached nearly $25 \mathrm{kPa}$ and $31 \mathrm{kPa}$ for initial gap size of 1 
The performance of a MR fluid in squeeze mode

and $2 \mathrm{~mm}$, respectively. Then, there is a plateau within the compressive stress remaining constant while the strain increased to values of about $16 \%\left(h_{0}=1 \mathrm{~mm}\right)$ and $8 \%\left(h_{0}=2\right.$ $\mathrm{mm}$ ). This phenomenon has not been reported by any previous researchers. In the final region, some aspects of the compressive stress began to increase again with further increased values of the compressive strain. The regions can be explained by assuming that there is relative movement between the particles and the carrier liquid in the MR fluid.

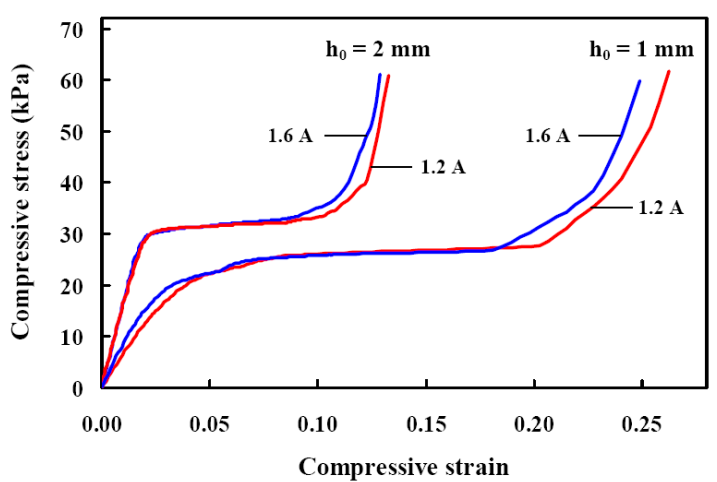

Figure 8. A larger scale of compressive stress versus compressive strain; at the beginning of the compression process when the applied current and initial gap size were set to 1.2 and 1.6 Amps, and 1 and $2 \mathrm{~mm}$, respectively.

Accordingly, by increasing the initial gap size, more particles are consumed inside the MR fluid. Even though the volume percent of particles is the same, the required compressive stress is distinctive. Therefore, the amount of particles is one of the factors that can affect the performance of the MR fluid. During the compression, the volume fraction of particle will increase because the liquid is assumed to be expelled from the MR fluid. Magnetic properties of the MR fluid will change as a result of this change in its composition. Consequently, the magnetic properties depend on the volume fraction of the particles in MR fluid. An assumption is made, which is supported by other researchers. It is that the magnetic properties of MR fluid can be increased by increasing the volume fraction (Simon et al 2001, Jolly et al 1999). Considering the average weight (w) percent particle is $85 \%$ 
The performance of a MR fluid in squeeze mode

with density ( $\rho$ ) $3.86 \mathrm{~g} / \mathrm{cc}$ (Lord 2007), and the average weight $(w)$ of carrier fluid (waterbased) is $15 \%$ with density $(\rho) 1 \mathrm{~g} / \mathrm{cc}$. Therefore, the volume percent for particle is given by:

$$
\% \text { Volume of particle }=\frac{[w / \rho]_{\text {particles }}}{[w / \rho]_{\text {water }}+[w / \rho]_{\text {particles }}}
$$

For instant, for $2 \mathrm{~mm}$ initial gap distance, the volume percent of particle is $59.54 \%$. At a displacement of $0.2 \mathrm{~mm}$ in initial gap distance, the volume fraction of particles will become $66.16 \%$. As the volume fraction increases, the magnetic field strength also increases which is described elsewhere by Jolly et al (1996). However, the process of expelling the liquid will continue until the process reaches the steady state. Higher magnetic field tends to retain more carrier liquid in the system as a MR fluid. In the prior experimental studies carried by Dang et al (2000) revealed that an increase in yield stress of $10-30$ volume $\% \mathrm{Fe}$ based fluids with increasing volume fraction. According to Jolly et al (1996), they illustrated that the saturation of iron particle is linearly proportional to the bulk saturation which saturates at 2.1 Tesla. For example, at certain value of magnetic intensity, if magnetic density for $59.54 \%$ volume of particle is 1.6 Tesla, so the magnetic density for $66.16 \%$ volume of particle is about $(66.16 / 59.14) \times 1.6=1.79$ Tesla. Therefore, by using this illustration, the magnetic properties of the tested MR fluid will increase when the volume fraction increases as shown in figure 9. On other hand, even though under a constant applied current, the magnetic field strength will increase as the gap closes because of the increase in volume percent of particle. 
The performance of a MR fluid in squeeze mode

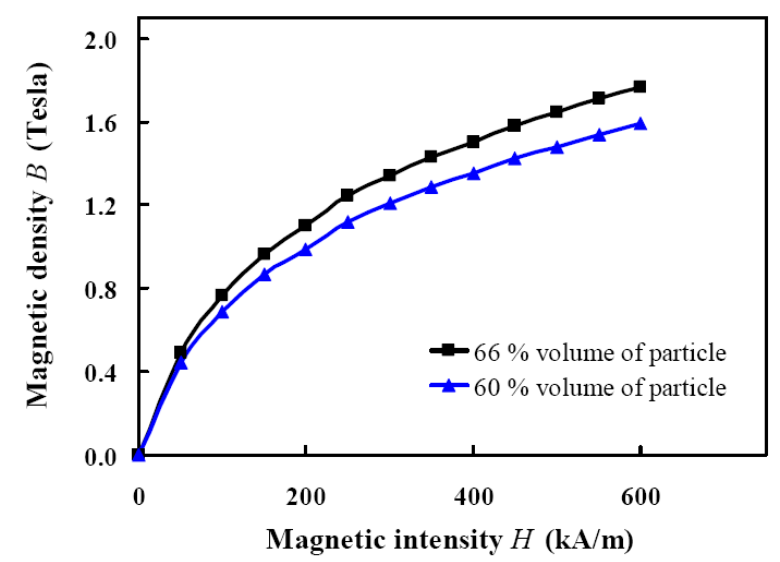

Figure 9. Magnetic induction curves of MR fluid for two different iron volume percents.

As further force is applied (third region); more deformation force is required to overcome the friction generated from the sliding between solid particles, and also to overcome the restriction of the solid particle movement due to the spatial constraint imposed by the surrounding particles. Most of the liquid expelled and the material became a solid-like material. The dry friction between the particles may greatly influence the static yield stress (Martin et al 1999). The applied stress represents the resistance of particles along magnetic flux direction under compression. Reducing the initial gap size between the two cylinders or increasing the applied current can increase the instantaneous magnetic field. The degree of deformation resistance is a function of the applied magnetic field strength. Once the magnetic field is applied, the particles of MR fluids are polarized and strongly interact with each other to form regular chains and columns along the field direction. These particle chains aggregated to form columns and to form even larger columns. Applying a pressure can extensively strengthen the stress. Comparable with compression in ER fluids, the mechanical property of MR fluid is determined by the chain/column strength and the deformation of the chains/columns under compression. These descriptive statements about 
The performance of a MR fluid in squeeze mode

chain strength under compression of MR fluid are credible but a great deal to describe the column size effects still yet to be studied.

\section{Conclusion}

The stress-strain relationships were obtained by applying the compression test on waterbased MR fluid. The compression process of MR fluid under different applied currents and initial gap sizes were presented. The experimental results revealed that the increase of compressive stress is subjected to the increase in compressive strain, where higher values can be achieved at higher currents. Furthermore, the compressive stress over compressive strain is greatly affected by the initial gap size. The amount of particles occupied inside the fluid can influence the compressive stress. For both initial gap sizes, the curves of compressive stress over compressive strain are derived, where the higher the initial gap size, the larger the compressive stress is. The curve is divided into three regions in order to describe the whole stress-strain relationship. For certain initial gap size, almost the same values are obtained at the first and second regions. Furthermore, the distance of compressive strain in the second region is dependent on the initial gap size. During the compression, in the second region, the compressive stress of MR fluid experienced a plateau progress nearly at $30 \mathrm{kPa}$ before proceeding to increase drastically. This phenomenon might be explained by the contribution of expelling process and the influence of the magnetic field strength. Hence, the compressive stress is not affected by the applied current. However, the curves showed a large variation in the third region as the applied current increased.

\section{References}

Ahn Y K, Ha J -Y and Yang B -S 2004 A new type controllable squeeze film damper using an electromagnet J. Vib. Acoust. 126 380-3

Carmignani C, Forte P and Rustighi E 2006 Design of a novel magneto-rheological squeeze-film damper Smart Mater. Struc. 15 164-70 
The performance of a MR fluid in squeeze mode

Dang A, Ooi L, Fales J and Stroeve P 2000 Yield stress measurements of magnetorheological fluids in tubes Ind. Eng. Chem. Res. 39 2269-74

Forte P Parterno M and Rustighi E 2004 A magnetorheological fluid damper for rotor applications Int. J. Rot. Mach. 10 175-82

Hagenbuchle M and Liu J 1997 Chain formation and chain dynamics in a dilute magnetorheological fluid Appl. Opt. 36 7664-71

Huang J, Zhang J Q, Yang Y and Wei Y Q 2002 analysis and design of a cylindrical magneto-rheological fluid brake J. Mater. Process. Tech. 129 559-62

Jolly M R, Bender J W and Carlson J D 1999 Properties and applications of commercial magnetorheological fluids J. Intell. Mater. Syst. Struct. 10 5-13

Jolly M R, Carlson J D and Munoz B C 1996 A model of the behaviour of magnetorheological materials Smart Mater. Struc. 5 607-14

Lord 2007, Lord Corporation, Lord Technical Data: MR Fluid MRF-241ES, http://www.lordfulfillment.com/upload/DS7013.pdf

Lukkarinen A and Kaski K 1998 Simulation studies of electrorheological fluids under shear, compression, and elongation loading J. Appl. Phys. 83 1717-25

Martin J E, Hill K M and Tigges C P 1999 Magnetic-field-induced optical transmittance in colloidal suspensions Phys. Rev. E 59 5676-92

Meeker D C 2006 FEMM, http://femm.foster-miller.net

Monkman G J 1995 The electrorheological effect under compressive stress J. Phys. D 28 588-93

Simon T M, Reitich F, Jolly M R, Ito K and Banks H T 2001 The effective magnetic properties of magnetorheological fluids Mathematical and Computer Modelling 33 273-84

Tian Y, Meng Y Mao H and Wen S 2002a Electrorheological fluid under elongation, compression, and shearing Phys. Rev. E 65 031507:1-5

2002b Mechanical property of electrorheological fluid under step compression J. Appl. Phys. 92 6875-9

Tian Y Wen S and Meng Y 2003 Compressions of electrorheological fluids under different initial gap

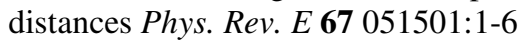

Wang J, Feng N, Meng G and Hahn E J 2006 Vibration control of rotor by squeeze film damper with magnetorheological fluid J. Intell. Mater. Syst. Struct. 17 353-7

Wang J, Meng G, Feng N and Hahn E J 2005 Dynamic performance and control of squeeze mode MR fluid damper-rotor system Smart Mater. Struc. 14 529-39

Yoo J -H and Wereley N M 2002 Design of a high-efficiency magnetorheological valve J. Intell. Mater. Syst. Struct. 13 679-85 